J. DIFFERENTIAL GEOMETRY

49 (1998) 411-435

\title{
PRECISELY INVARIANT COLLARS AND THE VOLUME OF HYPERBOLIC 3-FOLDS
}

\author{
F. W. GEHRING \& G. J. MARTIN
}

\begin{abstract}
We give a lower bound for the volume of any hyperbolic 3-orbifold which admits an embedded tubular neighborhood of a closed geodesic. This bound depends only on the radius of this neighbourhood and not on the length of the geodesic. In the Kleinian group uniformizing such a hyperbolic 3-fold, this yields a lower bound on the co-volume purely in terms of the radius of a precisely invariant collar of a loxodromic axis. As an application of these results we obtain substantial improvements in the known volume bounds of hyperbolic 3-manifolds and certain orbifolds. This paper forms part of our program to identify the minimal volume hyperbolic 3-fold.
\end{abstract}

\section{Introduction}

Hyperbolic 3-space is the set

$$
\mathbb{H}^{3}=\left\{\left(x_{1}, x_{2}, x_{3}\right) \in \mathbb{R}^{3}: x_{3}>0\right\}
$$

endowed with the complete Riemannian metric $d s=|d x| / x_{3}$ of constant curvature equal to -1 . We denote the hyperbolic distance in $\mathbb{H}^{3}$ by $\rho(\cdot, \cdot)$. A Kleinian group $G$ is a discrete nonelementary subgroup of $\operatorname{Isom}^{+}\left(\mathbb{H}^{3}\right)$, where $\operatorname{Isom}^{+}\left(\mathbb{H}^{3}\right)$ is the group of orientation preserving isometries. In this setting nonelementary means that the group $G$ is not virtually abelian. A hyperbolic 3 -orbifold $\mathcal{Q}$ is the orbit space of a Kleinian group. Thus

$$
\mathcal{Q}=\mathbb{H}^{3} / G
$$

Received May 30, 1997. Research supported in part by grants from the U. S. National Science Foundation and the N. Z. Marsden Fund.

1991 Mathematics Subject Classification. Primary 30F40, 20H10, 57N10. 
The orbit space is a hyperbolic 3-manifold if the group $G$ is torsion free.

The work of Thurston and others has shown that hyperbolic 3 -folds play a central role in low dimensional topology and geometry [30], [32]. Mostow's rigidity theorem [31] implies that volume is an important topological invariant of hyperbolic 3-folds. This paper is largely concerned with the problem of providing lower bounds for the volume of a hyperbolic 3-fold under various geometric constraints and forms part of our program to identify the minimal volume hyperbolic 3-fold. Additionally we obtain important geometric information about small volume hyperbolic $3-$ folds.

Using a formula due to Borel [4], Chinburg and Friedman showed that the smallest volume arithmetic hyperbolic 3 -fold is the orbit space of the Kleinian group $G_{(3,5,3)}$. This group is the $\mathbb{Z}_{2}$ extension of the orientation preserving elements in the tetrahedral reflection group with Coxeter diagram $3-5-3$ and co-volume $0.03905 \ldots$ [6]. More recently the smallest volume arithmetic hyperbolic 3-manifold was shown to be the Weeks manifold of volume $0.9427 \ldots$ [7].

Culler and Shalen have derived important lower bounds for the volumes of hyperbolic 3-manifolds which satisfy certain topological assumptions, for example, 0.34 if the first Betti number is at least 2, [9], $[10],[11]$. However the Weeks manifold, conjectured to be of smallest volume, has Betti number 0.

Until now only poor bounds were known for the volume of a general hyperbolic 3-manifold [29] and [19]. We derive here an estimate which, when combined with the results of Gabai, Meyerhoff and Thurston [14], yields a lower bound of $0.166 \ldots$ for the volume of an arbitrary hyperbolic 3-manifold.

We now summarize our main results. A collar of radius $t>0$ about a hyperbolic line $\ell$ is the set

$$
\mathcal{C}=\mathcal{C}(t)=\mathcal{C}(\ell, t)=\left\{x \in \mathbb{H}^{3}: \rho(x, \ell)<t\right\}
$$

$\mathcal{C}$ is precisely invariant with respect to $G$ if its translates under the group $G$ form a disjoint collection. See $\S 2$. Set

$$
\mathcal{V}(t)=\frac{\sqrt{3}}{2} \tanh (t) \cosh (2 t) \operatorname{arcsinh}^{2}(\sinh (t) / \cosh (2 t))
$$

Our first result is a volume estimate in terms of the radius of a precisely invariant collar. 
Theorem 1.4. If $G$ is a Kleinian group and if $\mathcal{C}(t)$ is a precisely invariant collar with respect to $G$, then

$$
\operatorname{Vol}\left(\mathbb{H}^{3} / G\right) \geq \frac{1}{2} \mathcal{V}(t)
$$

Moreover if $G$ is torsion free or if $G$ contains no primitive 2-torsion, then

$$
\operatorname{Vol}\left(\mathbb{H}^{3} / G\right) \geq 2 \mathcal{V}(t)
$$

We give specific conditions which guarantee a larger volume estimate. These involve the existence of a Klein 4-group whose finite fixed point lies in the boundary of a maximal precisely invariant collar.

There is currently substantial interest in hyperbolic 3-manifolds which contain an embedded tubular neighborhood of radius $(\log 3) / 2$. This is largely due to Gabai's version of the rigidity theorem [13].

Theorem 1.4 has the following consequence.

Corollary 1.7. If $M^{3}$ is a hyperbolic 3-manifold containing an embedded tubular neighborhood of radius $(\log 3) / 2$ about a closed geodesic, then

$$
\operatorname{Vol}\left(M^{3}\right) \geq \frac{5}{2 \sqrt{3}} \operatorname{arcsinh}^{2}\left(\frac{\sqrt{3}}{5}\right)=0.16668 \ldots
$$

Recently Gabai, Meyerhoff and N. Thurston announced a classification (via computer) of all closed finite volume hyperbolic 3-manifolds which do not contain such an embedded tubular neighborhood [14]. All those found have larger volume than the Weeks manifold. The next bound is a consequence of their classification and Corollary 1.7.

Theorem 1.9. If $M^{3}$ is a hyperbolic 3-manifold, then

$$
\operatorname{Vol}\left(M^{3}\right) \geq 0.16668 \ldots
$$

It is worth noting here that the previous best known lower bound for the volume of a hyperbolic 3-manifold was 0.00115 [19] and thus Theorem 1.9 represents a significant improvement.

The following result is an important consequence of Theorem 1.9.

Corollary 1.11. If $G$ is a Kleinian group of minimal co-volume, then $G$ contains no torsion free subgroup of index $n, n \leq 4$. 
An elliptic element in a Kleinian group is simple if its axis is precisely invariant. Corollary 1.11 and the results of [21] and [22] imply that a minimal co-volume Kleinian group is either $G_{(3,5,3)}$ or it is not torsion free and contains only simple elliptics of order $p, p \in\{2,3,4,5\}$. Using the results obtained herein we are able to eliminate the last two possibilities $p=4,5$.

Theorem 1.12. A Kleinian group of minimal co-volume does not contain a simple elliptic of order 4 or 5 .

As a final application of our results we give explicit lower bounds for the volume of hyperbolic 3-manifolds which admit a large cyclic symmetry. Such manifolds are termed fairly symmetric by Kuiper, and the Mennicke manifolds are conjectured to be extremal for this problem. Our methods give estimates off by a factor of about 2 from this conjecture and provide strong evidence for its validity.

\section{Precisely Invariant Collars}

Let $G$ be a Kleinian group. A subset $F$ of $\mathbb{H}^{3}$ is said to be precisely invariant with respect to $G$ if for each $g \in G$ either

$$
g(F)=F \quad \text { or } \quad g(F) \cap F=\emptyset .
$$

Given a set $F \subset \mathbb{H}^{3}$ we denote by $G_{F}$ the stabilizer of $F$ in $G$; that is,

$$
G_{F}=\{g \in G: g(F)=F\} .
$$

Every loxodromic or elliptic element $f$ in a Kleinian group fixes two points of $\overline{\mathbb{C}}=\partial \mathbb{H}^{3}$, and the closed hyperbolic line in $\mathbb{H}^{3}$ joining these two points is called the axis of $f$, denoted $\operatorname{ax}(f)$. Such an element $f$ translates along this axis by an amount $\tau(f) \geq 0$, the translation length, and rotates by an angle $\theta(f) \in(-\pi, \pi]$, the holonomy or rotation angle, about this axis.

A nonparabolic element $f \in G$ is simple if $\operatorname{ax}(f)$ is precisely invariant under $G$. The classification of the elementary discrete groups [3], [27] implies that an elliptic element of order $p \geq 7$ is simple. When $2 \leq p \leq 6$ an elliptic element in $G$ is simple unless it lies in a tetrahedral subgroup $(p=2,3)$, an octahedral subgroup $(p=2,3,4)$, an icosahedral subgroup $(p=2,3,5)$ or a parabolic subgroup, that is a group conjugate to an infinite group of euclidean isometries, $(p=2,3,4,6)$. Determining when there is a simple loxodromic axis in a Kleinian group is more difficult. 
However in a closed hyperbolic 3 -fold the shortest geodesic is simple and lifts to a simple loxodromic axis.

With each simple element of a Kleinian group there is an associated precisely invariant tubular neighborhood of its axis. Precisely invariant sets yield volume estimates because of the following elementary result.

Lemma 2.3. If $G$ is a Kleinian group and if $F \subset \mathbb{H}^{3}$ is precisely invariant with respect to $G$, then

$$
\operatorname{Vol}\left(\mathbb{H}^{3} / G\right) \geq \operatorname{Vol}\left(F / G_{F}\right)
$$

The point to this observation is that often $G_{F}$ is a far simpler group to understand than $G$, and with a judicious choice of $F$ the estimate need not be too bad.

The boundary of a collar $\mathcal{C}=\mathcal{C}(\ell, t)$

$$
\mathcal{S}=\mathcal{S}(\ell, t)=\partial \mathcal{C}
$$

is a hyperbolic cylinder. When $\{0, \infty\} \subset \ell, \mathcal{C}$ is a euclidean cone in $\mathbb{H}^{3}$. We let $\phi$ denote the solid angle at a vertex of this cone. Since Möbius transformations are conformal (angle preserving), $\phi$ is an invariant of $\mathcal{S}$, and $t$ and $\phi$ are related by the following formula.

Lemma 2.5. If $\mathcal{S}(\ell, t)$ is a hyperbolic cylinder and if $\phi$ is the solid angle, then

$$
\cos (\phi) \cosh (t)=1 \text {. }
$$

Proof. We may assume that $\ell$ has endpoints 0 and $\infty$. A geodesic from $\ell$ to $\mathcal{S}$ is

$$
\left\{\left(x_{1}, x_{2}, x_{3}\right)=(\sin (\varphi), 0, \cos (\varphi)): 0 \leq \varphi \leq \phi\right\} .
$$

Its hyperbolic length is

$$
t=\int_{0}^{\phi} \frac{d \varphi}{\cos (\varphi)}=\log (\sec (\phi)+\tan (\phi))
$$

from which we deduce $1=\cosh (t) \cos (\phi)$. q.e.d.

Next observe that the hyperbolic cylinder $\mathcal{S}(\ell, t)$ with the induced metric from $\mathbb{H}^{3}$ is isometric to a euclidean cylinder of radius $\sinh (t)$. We now collect together a few facts concerning hyperbolic cylinders. When $\ell$ has 0 and $\infty$ as its endpoints and $x=\left(x_{1}, x_{2}, x_{3}\right) \in \mathcal{S}$, we use the natural cylindrical coordinates $r=|x|$ and $\theta=\arctan \left(x_{2} / x_{1}\right)$. 
Lemma 2.7. Suppose that $\ell$ is a closed hyperbolic line containing $\{0, \infty\}$, that $t>0$, and that $\mathcal{S}=\mathcal{S}(\ell, t)$. Then $r, \theta$ are orthogonal coordinates on $\mathcal{S}$. If $E \subset \mathcal{S}$ is measurable, then the area of $E$ in the induced metric is

$$
\operatorname{Area}(E)=\cosh (t) \sinh (t) \int_{E} \frac{1}{r} d r d \theta
$$

Proof. It is clear that $r$ and $\theta$ form orthogonal coordinates. It therefore suffices to establish formula (2.8) for a small rectangle of the form $R=[a, b] \times\left[0, \theta_{0}\right]$. The length of the sides of this rectangle in the induced metric are

$$
\Delta \theta=\int_{0}^{\theta_{0}} \frac{r \sin (\phi) d \theta}{r \cos (\phi)}=\theta_{0} \tan (\phi)
$$

and

$$
\Delta r=\int_{a}^{b} \frac{d r}{r \cos (\phi)}=\frac{\log (b / a)}{\cos (\phi)}
$$

where $\phi$ is the solid angle subtended by $\mathcal{S}$. Therefore the area of this rectangle in the induced metric is the product of the lengths of the sides, namely

$$
\Delta \theta \Delta r=\theta_{0} \log (b / a) \frac{\tan (\phi)}{\cos (\phi)}=\frac{\tan (\phi)}{\cos (\phi)} \int_{R} \frac{1}{r} d r d \theta .
$$

Since Lemma 2.5 implies $\sin (\phi)=\tanh (t)$, the result follows. q.e.d.

Note that Lemma 2.7 could be rewritten in the form

$$
\operatorname{Area}(E)=\tanh (t) \int_{E} \frac{1}{x_{3}^{2}} r d r d \theta,
$$

and that $\Delta r$ and $\Delta \theta$ are the elements of arclength in the coordinate axes of the cylinder.

Suppose next that $G$ is a Kleinian group and that $\mathcal{C}$ is a precisely invariant collar whose boundary is a hyperbolic cylinder $\mathcal{S}$. Let $G_{\mathcal{C}}$ denote the stabilizer of $\mathcal{C}$. Then the stabilizer $G_{\mathcal{C}}$ of $\mathcal{C}$ acts on $\mathcal{S}$ as a properly discontinuous group of isometries in the induced metric. If $E \subset \mathcal{S}$ is a fundamental domain for the action of $G_{\mathcal{C}}$ on $\mathcal{S}$ and if $W$ is the set of all hyperbolic line segments perpendicular to $\ell$ with one endpoint on $\ell$ and the other in $E$, then $W$ is a fundamental domain for the action of $G_{\mathcal{C}}$ on $\mathcal{C}$. Therefore $\operatorname{Vol}(W)=\operatorname{Vol}\left(\mathcal{C} / G_{\mathcal{C}}\right)$. The next lemma relates the hyperbolic volume of $W$ and the area of $E$. 
Lemma 2.12. Let $\mathcal{S}=\mathcal{S}(\ell, t)$, and $E \subset \mathcal{S}$ be a measurable subset. Let $W$ be the union of all hyperbolic line segments which are perpendicular to $\ell$ and have one endpoint on $\ell$ and the other in $E$. Then

$$
\operatorname{Vol}(W)=\frac{1}{2} \tanh (t) \operatorname{Area}(E) .
$$

Proof. Let $\phi$ be the solid angle subtended by $\mathcal{S}$ and let $\{0, \infty\} \subset \ell$. We change from rectangular coordinates $\left(x_{1}, x_{2}, x_{3}\right)$ to spherical coordinates in $\mathbb{H}^{3}$ and deduce

$$
\operatorname{Vol}(W)=\int_{W} \frac{1}{x_{3}^{3}} d x_{1} d x_{2} d x_{3}=\int_{0}^{\phi} \int_{E(\varphi)} \frac{1}{r^{3} \cos ^{3}(\varphi)} r^{2} \sin (\varphi) d r d \theta d \varphi
$$

Here $E(\varphi)$ is the portion of $W$ meeting the cylinder about $\ell$ which subtends the angle $\varphi$. Now by construction the description of $E(\varphi)$ in $r, \theta$ coordinates does not depend on $\varphi$. Therefore

$$
\begin{aligned}
\operatorname{Vol}(W) & =\left(\int_{0}^{\phi} \frac{\sin (\varphi) d \varphi}{\cos ^{3}(\varphi)}\right)\left(\int_{E} \frac{1}{r} d r d \theta\right) \\
& =\frac{1}{2}\left(\frac{1}{\cos ^{2}(\phi)}-1\right) \frac{\operatorname{Area}(E)}{\cosh (t) \sinh (t)} \\
& =\frac{1}{2} \tanh (t) \operatorname{Area}(E)
\end{aligned}
$$

by Lemma 2.7 q.e.d.

We now turn to consider the problem of estimating the area of a fundamental domain for the group of euclidean isometries $G_{\mathcal{C}}$ acting on the cylinder $\mathcal{S}=\partial \mathcal{C}$.

\section{The shadow of a ball on a cylinder}

Let $\mathcal{S}=\mathcal{S}(\ell, t)$ be a hyperbolic cylinder. A hyperbolic ball $\mathbf{B}$ is tangent to $\mathcal{S}$ if $\partial \mathbf{B} \cap \mathcal{S}$ consists of a single point, the point of tangency, and if $\mathbf{B}$ does meet $\mathcal{C}(\ell, t)$. Let $W$ be the union of all hyperbolic line segments perpendicular to $\ell$, with one endpoint on $\ell$ and the other endpoint in $\mathbf{B}$. The shadow of $\mathbf{B}$ on $\mathcal{S}$ is

$$
\operatorname{shadow}(\mathbf{B})=\mathcal{S} \cap W \text {. }
$$

The area of the shadow on $\mathcal{S}$ depends only on the hyperbolic radius of B. 
In a subsequent section we shall show that the shadows of certain balls on the boundary of a precisely invariant collar form precisely invariant sets with respect to the stabilizer of the collar. Here we seek to compute the area of such a region. We do this by finding the parametric equation of the region in euclidean coordinates in the induced metric. However instead of computing the area it is more profitable to produce an inscribed ellipse in the region. This is because, although we give up some area by looking at a slightly smaller region, it is more than recouped after using some elementary facts about the density of ellipse packings in the plane.

Theorem 3.2. Let $\mathcal{S}=\mathcal{S}(\ell, t)$ be a cylinder and let $\mathbf{B}$ be a ball of hyperbolic radius $t>0$ tangent to $\mathcal{S}$. Then the shadow of $\mathbf{B}$ on $\mathcal{S}$ is congruent to the planar region bounded by the curve with parametric equations

$$
\begin{aligned}
x & =u \cosh (t), \\
|y| & =\sinh (t) \arcsin \left(\operatorname{coth}(2 t) \sqrt{\cosh ^{2}(a)-\cosh ^{2}(u)}\right) \\
\text { for }-a \leq u \leq & \leq \text {, where }
\end{aligned}
$$

$$
a=\operatorname{arcsinh}(\sinh (t) / \cosh (2 t)) .
$$

Proof. We may assume that $\{0, \infty\} \subset \ell$, and that the hyperbolic center $x_{0}$ of $\mathbf{B}$ lies on the unit sphere and in the $\left\{x_{2}=0\right\}$ plane, denoted $\Pi$. Now $\Pi$ contains $\ell$, the point $x_{0}$ and the euclidean center of $\mathbf{B}$. Let $s>1$ be the euclidean radius of the sphere about the origin tangent to $\mathbf{B}$, let $\phi$ be the angle subtended by $\mathcal{S}$ and let $\alpha$ be the angle subtended between the line $\ell$ and the ray from the origin through $x_{0}$. We describe the region parametrically in terms of the variable $r=|x|$.

Consider the Lambert quadrilateral bounded by the four hyperbolic lines consisting of $\ell$, the hyperbolic line through $x_{0}$ and perpendicular to $\ell$, either of the hyperbolic lines perpendicular to $\ell$ lying in $\Pi$ and tangent to the circle $\Pi \cap \mathbf{B}$ and the fourth side consists of the hyperbolic line through $x_{0}$ and perpendicular to this latter side (part of which then forms a hyperbolic radius of $\mathbf{B}$ ). The only angle which is not right is that subtended at $x_{0}$. We want to find the euclidean length of the side of length $a=\log (s)$ in $\ell$. The length of the opposite side is $t$ and $\rho\left(x_{0}, \ell\right)=2 t$. 


\section{Lemma 3.4.}

$$
\sinh (a)=\frac{\sinh (t)}{\cosh (2 t)}
$$

Proof. From the point to line formula (7.20.4) of [3] we have

$$
\sinh (t)=\frac{s^{2}-1}{2 s \cos (\alpha)}
$$

Next

$$
\sinh (a)=\frac{s^{2}-1}{2 s}
$$

and hence by $(2.6)$

$$
\cosh (2 t)=\frac{1}{\cos (\alpha)}=\frac{\sinh (t)}{\sinh (a)}
$$

q.e.d.

Next for each fixed $r \in[1 / s, s]$ we need to compute how the angle $\theta$ varies. Geometrically we see that if $\theta_{r}$ is half the spherical diameter of the cap $\mathbb{S}^{2}(0, r) \cap \mathbf{B}$, then $\theta \in\left[-\theta_{r}, \theta_{r}\right]$.

Lemma 3.6. If $\eta$ is the angle subtended by $\ell$ and the ray from 0 through the euclidean center of $\mathbf{B}$, then

$$
\tan (\eta)=2 \sinh (t)
$$

Proof. The euclidean radius of $\mathbf{B}$ is $\left(s-s^{-1}\right) / 2=\sinh (a)$. Since $\phi$ is the angle subtended by $\mathcal{S}, \cos (\phi)=\operatorname{sech}(t)$ by Lemma 2.5 , that is $\tan (\phi)=\sinh (t)$. Let $\beta=\eta-\phi$. Clearly $\tan (\beta)=\sinh (a)$. Since $\tan (\eta)=\tan (\phi+\beta),(3.7)$ follows from the angle sum formula for tangent and the identity $\cosh (2 t)=\cosh ^{2}(t)+\sinh ^{2}(t)$. q.e.d.

Next consider the situation in the plane $\Pi$. The euclidean center of $\mathbf{B}$ has coordinates $(\cosh (a) \sin (\eta), \cosh (a) \cos (\eta))$, while its euclidean radius is $(s-1 / s) / 2=\sinh (a)$. Let $\psi$ denote the angle between $\ell$ and the ray from the origin through the point $(r \sin (\psi), r \cos (\psi))$ of $\mathbf{B} \cap \mathbb{S}^{2}(0, r) \cap \Pi$ farthest from $\ell$. The euclidean law of cosines implies

$$
\frac{r+1 / r}{2 \cosh (a)}=\cos (\psi-\eta)
$$


Set

$$
r=e^{u}, \quad 0 \leq u \leq a,
$$

whence

$$
\frac{\cosh (u)}{\cosh (a)}=\cos (\psi-\eta)
$$

Then $\psi-\eta$ is the spherical radius of the disk $\mathbf{B} \cap \mathbb{S}^{2}(0, r)$. The angle we want is $2 \theta_{r}$ subtended at $\ell \cap \mathbb{S}^{2}(0, r)$. The angle between $\ell$ and the ray through the origin and the center of this disk is $\eta$. The spherical law of sines implies that

$$
\sin \left(\theta_{r}\right)=\frac{\sin (\psi-\eta)}{\sin (\eta)}
$$

from which we deduce

$$
\sin \left(\theta_{r}\right)=\frac{1}{\sin (\eta)} \sqrt{1-\frac{\cosh ^{2}(u)}{\cosh ^{2}(a)}} .
$$

The following lemma simplifies the formula above.

Lemma 3.13. With the notation above we have

$$
\sin (\eta) \cosh (a)=\tanh (2 t)
$$

Proof. First note that

$$
\cosh ^{2}(a)=\sinh ^{2}(a)+1=\left(\frac{\sinh (t)}{2 \sinh ^{2}(t)+1}\right)^{2}+1,
$$

and that

$$
\frac{1}{\sin ^{2}(\eta)}=\cot ^{2}(\eta)+1=\frac{1}{4 \sinh ^{2}(t)}+1
$$

Therefore

$$
\cosh ^{2}(a) \sin ^{2}(\eta)=\frac{4 \sinh ^{2}(t)\left(\sinh ^{2}(t)+1\right)}{\left(2 \sinh ^{2}(t)+1\right)^{2}}=\tanh ^{2}(2 t) .
$$

q.e.d. 
We can now give a parametric equation of the shadow. The line segment

$$
[1 / s, s]=\left\{\left(x_{1}, x_{2}, x_{3}\right)=(r \sin (\phi), 0, r \cos (\phi)): s^{-1} \leq r \leq s\right\}
$$

is a geodesic in the induced metric on the cylinder of length

$$
\int_{1 / s}^{s} \frac{d r}{r \cos (\phi)}=2 a \cosh (t)
$$

Next, the arcs of circles given by the intersection of planes $\left\{x_{3}=\right.$ $r \cos (\phi)\}$ with the cylinder $\mathcal{S}$ are geodesics in the induced metric which are perpendicular to the line segment $[1 / u, u]$. The angle subtended by the boundary of the shadow at height $r \cos (\phi)$ is $2 \theta_{r}$. The length of the arc $\left\{0<\theta<\theta_{r}\right\}$ in the induced metric is $\theta_{r} \tan (\phi)=\theta_{r} \sinh (t)$. Therefore as $r$ varies from 1 to $s$ we have $u$ varying from 0 to $a$, and the perpendicular distance in the induced metric from the line segment $[1 / s, s]$ to the boundary point of the shadow above $r$ is

$$
\sinh (t) \arcsin \left(\frac{1}{\sin (\eta)} \sqrt{1-\frac{\cosh ^{2}(u)}{\cosh ^{2}(a)}}\right) .
$$

Therefore the region bounded by the shadow is congruent to the planar region with parametric equations

$x=u \cosh (t), \quad y= \pm \sinh (t) \arcsin \left(\operatorname{coth}(2 t) \sqrt{\cosh ^{2}(a)-\cosh ^{2}(u)}\right)$.

as required. q.e.d.

Lemma 3.18. For $-1 \leq u \leq 1$,

$$
\arcsin \left(\operatorname{coth}(2 t) \sqrt{\cosh ^{2}(a)-\cosh ^{2}(a u)}\right) \geq a \operatorname{coth}(2 t) \sqrt{1-u^{2}}
$$

Proof. Clearly $\arcsin (v) \geq v$ for $v \in[0,1]$. The result then follows from the identity

$$
\cosh ^{2}(a)-\cosh ^{2}(a u) \geq a^{2}\left(1-u^{2}\right)
$$

which holds for all $a>0$ and $-1 \leq u \leq 1$, and is easily verified by calculus. q.e.d. 
Remark. As

$$
\begin{aligned}
0 & \leq \operatorname{coth}(2 t) \sqrt{\cosh ^{2}(a)-\cosh ^{2}(a u)} \\
& \leq \operatorname{coth}(2 t) \sinh (a) \\
& =1 / 2 \operatorname{sech}(t) \leq 1 / 2
\end{aligned}
$$

and the actual angle varies between 0 and $\pi / 6$, we have not given up too much in the estimate of Lemma 3.18.

Corollary 3.21. Let $\mathcal{S}=\mathcal{S}(\ell, t)$ be a cylinder, and $\mathbf{B}$ a ball of hyperbolic radius $t$ tangent to $\mathcal{S}$. Then the shadow of $\mathbf{B}$ on $\mathcal{S}$ contains a region congruent to an ellipse with parametric equations

$$
\left.\begin{array}{l}
x=a \cosh (t) \sin (\theta) \\
y=a \sinh (t) \operatorname{coth}(2 t) \cos (\theta))
\end{array}\right\} \quad-\pi \leq \theta \leq \pi,
$$

where $a=\operatorname{arcsinh}(\sinh (t) / \cosh (2 t))$.

Proof. This follows directly from the estimate of Lemma 3.18 after replacing $u$ by $\sin (\theta)$. q.e.d.

Remark. Notice that the ellipse contained in the shadow of any other ball of radius $t$ tangent to $\mathcal{C}$ is parallel to that given in equation (3.22) in the sense that if we lift to the universal cover the two ellipses are obtained one from the other by a translation.

Theorem 3.23. Let $G$ be a Kleinian group and let $\mathcal{C}=\mathcal{C}(\ell, t)$ be $a$ precisely invariant collar. Suppose that there is a collection of balls $\mathbf{B}_{i}$, $i=1,2, \ldots, n$ each of hyperbolic radius $t>0$ which are all tangent to $\mathcal{C}$, each ball is precisely invariant under $G_{\mathcal{C}}$ and for all $g \in G_{\mathcal{C}}$ we have $g\left(\mathbf{B}_{i}\right) \neq \mathbf{B}_{j}$ when $i \neq j$. Let $d_{i}$ be the order of the stabilizer of the ball $\mathbf{B}_{i}$ in $G_{\mathcal{C}}$. Then $d_{i} \in\{1,2\}$ and there is a fundamental domain $E \subset \partial \mathcal{C}$ of area

$$
\text { Area }(E) \geq \sqrt{3} \cosh (2 t) \operatorname{arcsinh}^{2}(\sinh (t) / \cosh (2 t)) \sum_{i=1}^{n} \frac{1}{d_{i}}
$$

for the group $G_{\mathcal{C}}$ acting on $\mathcal{C}$.

Proof. We may suppose that $\{0, \infty\} \subset \ell$. We view things in the induced metric of $\mathcal{S}=\partial \mathcal{C}$. Here the shadow of each ball $\mathbf{B}_{i}$ contains an ellipse $E_{i}$ whose parametric equations are given by (3.22). The ellipse $E_{i}$ is precisely invariant with respect to $G_{\mathcal{C}}$ and has stabilizer of order $d_{i} \in\{1,2\}$. To see this, note that if $g \in G_{\mathcal{C}}$, and $g\left(E_{i}\right) \cap E_{i} \neq \emptyset$, 
then $g\left(\mathbf{B}_{i}\right)=\mathbf{B}_{i}$ and hence $g$ is a primitive elliptic of order two, whose axis meets $\ell$ perpendicularly and passes through the point of tangency of $\mathbf{B}_{i}$. This point is the center of $E_{i}$ and it follows that $g\left(E_{i}\right)=E_{i}$. Next, the translates of $\bigcup_{i=1}^{n} E_{i}$ under the group $G_{\mathcal{C}}$ give a packing of the euclidean cylinder $\mathcal{S}$ by disjoint congruent ellipses. In view of the preceding Remark we see that in the universal cover (the euclidean plane) all these ellipses are equivalent via translations. After an affine area preserving change of coordinates we obtain a packing by round disks. The densest such packing is the hexagonal packing of density $\frac{2 \sqrt{3}}{\pi}$. This then also bounds the densest packing by disjoint congruent ellipses on $\mathcal{S}$. Each $E_{i}$ therefore contributes $2 \sqrt{3} \operatorname{Area}\left(E_{i}\right) /\left(\pi d_{i}\right)$ to the area of a fundamental region for the action of $G_{\mathcal{C}}$ on $\mathcal{C}$. This proves the result. q.e.d.

We next define the function

$$
\mathcal{V}(t)=\frac{\sqrt{3}}{2} \tanh (t) \cosh (2 t) \operatorname{arcsinh}^{2}(\sinh (t) / \cosh (2 t)) .
$$

We leave the verification of the following elementary lemma to the reader.

Lemma 3.25. $\mathcal{V}(t)$ is increasing on $[0, \infty]$ and

$$
\lim _{t \rightarrow \infty} \mathcal{V}(t)=\frac{\sqrt{3}}{4}
$$

The following theorem is now a direct consequence of Lemma 2.12 and Theorem 3.23.

Theorem 3.27. Let $G$ be a Kleinian group, and $\mathcal{C}=\mathcal{C}(\ell, t)$ a precisely invariant collar. Suppose that there is a collection of balls $\mathbf{B}_{i}$, $i=1,2, \ldots, n$ such that:

1. each ball has hyperbolic radius $t$,

2. each ball is tangent to $\mathcal{C}$ and precisely invariant with respect to $G_{\mathcal{C}}$,

3. for all $g \in G_{\mathcal{C}}$ we have $g\left(\mathbf{B}_{i}\right) \neq \mathbf{B}_{j}$ when $i \neq j$,

4. order of the stabilizer of the ball $\mathbf{B}_{i}$ in $G_{\mathcal{C}}$ is $d_{i}$.

Then $d_{i} \in\{1,2\}$ and

$$
\operatorname{Vol}(\mathcal{C} / G) \geq \mathcal{V}(t) \sum_{i=1}^{n} \frac{1}{d_{i}}
$$




\section{Maximal collars and tangent balls}

In order to use Theorem 3.27 to produce lower bounds for the covolume of Kleinian groups we need to establish the existence of precisely invariant tangent balls. We will also want to identify some interesting properties of the stabilizers of these balls.

Let $G$ be a Kleinian group, and $\mathcal{C}$ a precisely invariant collar with respect to $G$. We say that $\mathcal{C}$ is maximal if there is no collar of larger radius about $\ell$ which is also precisely invariant with respect to $G$. It is easy to see that if $\mathcal{C}$ is precisely invariant under a Kleinian group $G$, then $\mathcal{C}$ lies inside a maximal precisely invariant collar. What is not immediate is the following lemma.

Lemma 4.1. Let $G$ be a Kleinian group, and $\mathcal{C}=\mathcal{C}(\ell, t)$ be a maximal precisely invariant collar. Suppose that $G_{\mathcal{C}}$ is infinite. Then there is $g \in G$ and a unique point $x_{0} \in \partial \mathcal{C}$ such that

$$
g(\overline{\mathcal{C}}) \cap \overline{\mathcal{C}}=\left\{x_{0}\right\}
$$

Proof. Assume that $\{0, \infty\} \subset \ell$. As $G_{\mathcal{C}}$ is infinite there is a primitive loxodromic element $h \in G_{\mathcal{C}}$ of translation length $\tau>0$. Let $B$ be the ball centered at $(0,0,1) \in \mathbb{H}^{3}$ of hyperbolic radius $\tau+3 t$. Notice that if $x \in \mathbb{H}^{3}$ is any point with $\rho(x, \ell)<3 t$, then an application of the triangle inequality, and the fact that $h(\ell)=\ell$ shows there is an $n \in \mathbb{Z}$ such that $h^{n}(x) \in B$. As $\mathcal{C}$ is maximal, there is a sequence $\left\{g_{j}\right\} \in G \backslash G_{\mathcal{C}}$ such that

$$
\rho\left(\mathcal{C}, g_{j}(\mathcal{C})\right) \rightarrow 0 \quad \text { as } \quad j \rightarrow \infty
$$

If for some $j, \rho\left(\mathcal{C}, g_{j}(\mathcal{C})\right)=0$, then the result follows from elementary geometric considerations and the fact that $\mathcal{C}$ is geodesically strictly convex. Thus we assume otherwise. Relabeling and passing to a subsequence we assume

$$
0<\rho\left(\mathcal{C}, g_{j}(\mathcal{C})\right)<\frac{1}{j}
$$

Now let $y_{j} \in g_{j}(\ell)$ be the closest point of $g_{j}(\ell)$ to $\ell$. So for $j$ sufficiently large,

$$
\rho\left(y_{j}, \ell\right)<2 t+\frac{1}{j}<3 t .
$$


Then there is $m \in \mathbb{Z}$ such that $h^{m}\left(y_{j}\right) \in B$. There is also $n \in \mathbb{Z}$ such that $h^{n}\left(g_{j}^{-1}\left(y_{j}\right)\right) \in B$ from which we deduce that $h^{m} g_{j} h^{-n}(B) \cap B \neq \emptyset$ for all sufficiently large $j$.

As $G$ is discrete it acts properly discontinuously in $\mathbb{H}^{3}$. This contradicts the fact that

$$
\rho\left(g_{j}(\mathcal{C}), \mathcal{C}\right)=\rho\left(h^{m} g_{j} h^{-n}(\mathcal{C}), \mathcal{C}\right)=\rho\left(y_{j}, \ell\right)-2 t,
$$

which implies by the above that infinitely many of the images $h^{m} g_{j} h^{-n}(B)$ are distinct. q.e.d.

We are now in a position to establish the existence of the precisely invariant tangent balls.

Lemma 4.6. Given $\mathcal{C}, g$ and $x_{0}$ as above, let $\mathbf{B}_{1}$ be the ball tangent to $\mathcal{C}$ at $x_{0}$ of hyperbolic radius $t$ (the same as $\mathcal{C}$ ). Then $\mathbf{B}_{1}$ is precisely invariant under $G_{\mathcal{C}}$. If $g \in G_{\mathcal{C}}$ and $g\left(\mathbf{B}_{1}\right) \cap \mathbf{B}_{1} \neq \emptyset$, then $g$ is the identity or a primitive elliptic of order two fixing $x_{0}$, interchanging 0 and $\infty$, whose axis passes through $x_{0}$ and meets $\ell$ perpendicularly.

Proof. Observe that $\mathbf{B}_{1} \subset g(\mathcal{C})$. If $h\left(\mathbf{B}_{1}\right) \cap \mathbf{B}_{1} \neq \emptyset$ for some $h \in G_{\mathcal{C}} \backslash\{I d\}$, then $h(g(\mathcal{C})) \cap g(\mathcal{C}) \neq \emptyset$. As $\mathcal{C}$ is precisely invariant, so too is $g(\mathcal{C})$ and we deduce that $h(g(\mathcal{C}))=g(\mathcal{C})$. Also $h(\mathcal{C})=\mathcal{C}$ and these two facts together imply $h\left(x_{0}\right)=x_{0}$. If $h$ is not the identity, $h$ is easily seen to be a primitive elliptic element of order two which must interchange 0 and $\infty$. q.e.d.

Thus in the situation above the ball $\mathbf{B}_{1}$ and its orbit under the group $G_{\mathcal{C}}$ (that is the collection $\left\{h(\mathbf{B}): h \in G_{\mathcal{C}}\right\}$ ) form a disjoint collection of balls all tangent to $\mathcal{C}$. For the case that there is a precisely invariant horoball in a torsion free group, there is a nice trick due to Adams [1] which enables one to produce another ball $\mathbf{B}_{2}$ of the same radius again tangent to the precisely invariant horosphere but disjoint from the orbit of $\mathbf{B}_{1}$. Here is a version of that trick in our setting.

Lemma 4.7. Let $\mathcal{C}, g, x_{o}$ and $\mathbf{B}_{1}$ be as above. Suppose there is no primitive elliptic element of order two in $G$ whose axis passes through $x_{0}$ and is tangent to $\partial \mathcal{C}$. Then the ball $\mathbf{B}_{2}$ tangent to $\mathcal{C}$ at $g^{-1}\left(x_{0}\right)$ and of radius $t$ is also precisely invariant, and for no $h \in G_{\mathcal{C}}$ is it true that $h\left(\mathbf{B}_{2}\right) \cap \mathbf{B}_{1} \neq \emptyset$.

Proof. Suppose that $g$ is not the elliptic of order two described above. Let $\mathbf{B}_{2}$ be as above, so that

$$
\mathbf{B}_{2} \subset g^{-1}(\mathcal{C}) \neq g(\mathcal{C}),
$$


as $\mathcal{C}$ and $g(\mathcal{C})$ are tangent. Notice that the precise invariance of $\mathcal{C}$ implies that if there is $h \in G_{\mathcal{C}}$ such that $h\left(\mathbf{B}_{2}\right) \cap \mathbf{B}_{1} \neq \emptyset$, then $h\left(\mathbf{B}_{2}\right)=\mathbf{B}_{1}$. Moreover, $g^{-1} h g^{-1}: g\left(\mathbf{B}_{2}\right) \subset \mathcal{C} \rightarrow \mathcal{C}$. Precise invariance yields therefore that $g^{-1} h g^{-1}(\mathcal{C})=\mathcal{C}$ and hence $h g^{-1}\left(x_{0}\right)=x_{0}$. Let $f=h g^{-1}$. Then $f(\mathcal{C})=g(\mathcal{C})$ and $f\left(x_{0}\right)=x_{0}$. As $\mathcal{C}$ and $g(\mathcal{C})$ are tangent, it follows that $f$ is a primitive elliptic of order two whose axis meets $x_{0}$ and is tangent to $\partial \mathcal{C}$. A contradiction. q.e.d.

Lemma 4.8. If $G$ is a Kleinian group with $\operatorname{Vol}\left(\mathbb{H}^{3} / G\right)<\infty$ and $\mathcal{C}$ is a precisely invariant collar, then $G_{\mathcal{C}}$ is infinite.

Proof. The hyperbolic volume of a collar of positive radius is infinite. If it is precisely invariant, then Lemma 2.3 implies that $G_{\mathcal{C}}$ is infinite. q.e.d.

In order to make effective use of Theorem 3.27 we need to say something about the magnitude of the sum $\sum 1 / d_{i}$ of the reciprocals of the order of the stabilizers of the tangent balls. Lemma 4.6 above says that the order of the stabilizer of a tangent ball is either one or two, and it is two if and only if there is a primitive elliptic axis of order two passing through the point of tangency and meeting $\ell$ perpendicularly. Lemma 4.7 implies further that there are two distinct tangent balls unless there is a primitive elliptic of order two whose axis passes through the point of tangency and is tangent to the boundary of the collar. Thus $\sum 1 / d_{i}=1 / 2$ only if there is a pair of primitive elliptic elements of order two whose axes meet orthogonally, one axis tangent to $\mathcal{C}$ and the other perpendicular. Two such elliptics generate a Klein 4 -group, $K_{4}$, and the point of $\mathbb{H}^{3}$ stabilized by this finite group is the point of tangency of the ball arising from the maximal collar. Thus if there is no Klein 4-group fixing a point of the boundary of the collar, we see that $\sum 1 / d_{i} \geq 1$ while if there are no primitive elliptics of order two, then $\sum 1 / d_{i} \geq 2$. We have proved the following theorem. (Recall $\mathcal{V}(t)$ is defined at (3.24).)

Theorem 4.9. Suppose that $G$ is a Kleinian group and that $\mathcal{C}=$ $\mathcal{C}(\ell, t)$ is a maximal precisely invariant collar. Then

$$
\operatorname{Vol}\left(\mathbb{H}^{3} / G\right) \geq \frac{1}{2} \mathcal{V}(t)
$$

Furthermore, we have the following:

1. If there is no Klein 4-group stabilising a point on the boundary of 
$\mathcal{C}$, then

$$
\operatorname{Vol}\left(\mathbb{H}^{3} / G\right) \geq \mathcal{V}(t)
$$

2. If there is no primitive elliptic element of order two whose axis meets the boundary of $\mathcal{C}$, then

$$
\operatorname{Vol}\left(\mathbb{H}^{3} / G\right) \geq 2 \mathcal{V}(t)
$$

3. If $G$ is torsion free, or $G$ contains no primitive $2-$ torsion, then

$$
\operatorname{Vol}\left(\mathbb{H}^{3} / G\right) \geq 2 \mathcal{V}(t)
$$

\section{Applications}

In this section we give some applications of our results to questions concerning the volume of hyperbolic 3 -folds.

\subsection{Manifolds}

Because of the interest in Gabai's topological rigidity theorem for hyperbolic 3-manifolds [13], there has been quite a bit of interest in hyperbolic 3-manifolds which admit a precisely invariant collar of radius $(\log 3) / 2$. This situation also arose in the work of Culler-Shalen [9], [10], [11]. Geometrically the assumption means that in the manifold there is an embedded tubular neighbourhood of radius $(\log 3) / 2$ about a closed geodesic without any apriori assumption about the length of this closed geodesic. Recently D. Gabai, R. Meyerhoff and N. Thurston announced a classification (via computer) of all closed finite volume hyperbolic 3manifolds which do not admit such a precisely invariant collar of radius $(\log 3) / 2$ [14]. Those found all have larger volume than the conjectured minimum. That is of the Weeks manifold, a hyperbolic manifold with volume about $0.9427 \ldots$ As a direct consequence of Theorem 4.9 and Lemma 3.25 we obtain the following lower bound for the volume of a complete hyperbolic 3-manifold.

Theorem 5.1. Suppose that $M^{3}$ is a hyperbolic 3-manifold with an embedded tubular neighourhood of radius $(\log 3) / 2$ about a closed geodesic. Then

$$
\operatorname{Vol}\left(M^{3}\right) \geq 0.16668 \ldots
$$


Proof. The uniformizing Kleinian group $G$ is torsion free and has a precisely invariant collar of radius at least $(\log 3) / 2$. Therefore by Theorem 4.9 we have

$$
\operatorname{Vol}\left(\mathbb{H}^{3} / G\right) \geq 2 \mathcal{V}((\log 3) / 2)>0.16668 \ldots,
$$

since by Lemma $3.25, \mathcal{V}(t)$ is increasing. q.e.d.

In view of the announcement [14], the bound 0.16668 would appear to be a lower bound for the volume of any hyperbolic 3-manifold. This bound is two orders of magnitude larger than all previous bounds; see [19], [29].

Corollary 5.4. If $M^{3}$ is a hyperbolic 3-manifold, then

$$
\operatorname{Vol}\left(M^{3}\right) \geq 0.16668 \ldots .
$$

Proof. It only remains to observe that if $M^{3}$ is not closed, then $\operatorname{Vol}\left(M^{3}\right) \geq \sqrt{3} / 4$ by $[1]$. q.e.d.

In particular the above bound for the volume of a hyperbolic manifold exceeds a known bound for the minimal volume of a hyperbolic 3 -orbifold. We conclude that the minimal co-volume Kleinian group is not torsion free.

Corollary 5.6. Let $G$ be a Kleinian group of minimal co-volume. Then $G$ is not torsion free and contains no torsion free subgroup of order 4 or less.

\subsection{Simple elliptic elements}

Recall that an elliptic element $g$ of a Kleinian group $G$ is called simple if $\operatorname{ax}(g)$ is precisely invariant with respect to $G$. In this subsection we show that a Kleinian group of minimal co-volume cannot contain a simple elliptic of order $p, p \geq 4$. Previously we have shown that a Kleinian group of minimal co-volume does not contain any elliptics of order six or more [21] and here, as an application of Theorem 4.9, we will give a new proof of this fact as well.

We first recall that the classification of the elementary Kleinian groups [3], [27] implies that an elliptic of order $p \geq 7$ is simple. Next in $[20]$ we showed that such an elliptic element has a precisely invariant collar of radius

$$
r_{p}=\operatorname{arccosh}\left(\frac{1}{2 \sin (\pi / p)}\right), \quad p \geq 7,
$$


Furthermore we showed in that paper that a simple elliptic element of order 6 has a precisely invariant collar of radius

$$
r_{6}=0.658 \ldots
$$

Notice that $r_{p}$ is increasing with $p$ for $p \geq 7$ and that $r_{6}>r_{7}$. We now have the following theorem, cf. [21].

Theorem 5.9. Let $G$ be a Kleinian group containing an elliptic element of order $p \geq 6$. Then

$$
\operatorname{Vol}\left(\mathbb{H}^{3} / G\right) \geq \mathcal{V}\left(r_{p}\right) / 2 \geq 0.04
$$

Proof. If the elliptic element is simple, then the result follows. If the elliptic is not simple, then $p=6$ and the quotient is noncompact and therefore, by a result of Meyerhoff [28], has volume at least $0.081 \ldots$.

q.e.d.

We next extend this result to include simple elliptics of orders 4 and 5. We begin by observing the following consequence of Theorem 4.9.

Lemma 5.11. Suppose that $G$ is a Kleinian group of minimal covolume and that $\mathcal{C}(\ell, t)$ is a precisely invariant collar with respect to $G$. Then $t<0.533$.

Proof. It suffices to observe that $\mathcal{V}(.533) / 2>0.03906$ which is larger than the co-volume of $G_{(3,5,3)}$. $\quad$ q.e.d.

The following tables tabulate in increasing order the possible distances between elliptic axes of order 4 (Table 1) and order 5 (Table 2) in a discrete group. Also included is information on the arithmeticity of the Kleinian group $G$ generated by the elliptic and its closest translate and the co-volume of the minimal volume arithmetic Kleinian group in which $G$ embeds. These tables are extracted from [23]. 
Table 1 - Groups $\boldsymbol{G}_{\mathbf{4}, \boldsymbol{i}} \quad \delta_{4, i} \leq 0.614$

\begin{tabular}{|c|c|c|c|c|}
\hline$i$ & $\delta_{4, i}$ & Simple Axis & Arithmetic & Minimal Co-volume \\
\hline 1 & 0 & No & Yes & -- \\
\hline 2 & .4157 & Yes & Yes & $0.1268 \ldots$ \\
\hline 3 & .4269 & No & Yes & $0.1374 \ldots$ \\
\hline 4 & .4406 & No & Yes & $0.2289 \ldots$ \\
\hline 5 & .5049 & Yes & Yes & $0.2968 \ldots$ \\
\hline 6 & .5225 & Yes & Yes & $0.2968 \ldots$ \\
\hline 7 & .5297 & Yes & Yes & $0.0661 \ldots$ \\
\hline 8 & .5297 & No & Yes & $0.0661 \ldots$ \\
\hline 9 & .5306 & No & Yes & $0.1526 \ldots$ \\
\hline 10 & .5306 & Yes & Yes & Fuchsian \\
\hline 11 & .5306 & Yes & No & $0.0717 \ldots$ \\
\hline 12 & .5345 & No & Yes & $0.4475 \ldots$ \\
\hline 13 & .6130 & No & Yes & $0.3475 \ldots$ \\
\hline
\end{tabular}

Table 2 - Groups $\boldsymbol{G}_{\mathbf{5}, i} \quad \delta_{5, i} \leq 0.63$

\begin{tabular}{|c|c|c|c|c|}
\hline$i$ & $\delta_{5, i}$ & Simple Axis & Arithmetic & Minimal Co-volume \\
\hline 1 & 0 & No & Yes & -- \\
\hline 2 & .4568 & No & Yes & $0.0933 \ldots$ \\
\hline 3 & .5306 & No & Yes & $0.0390 \ldots$ \\
\hline 4 & .6097 & Yes & Yes & $0.0390 \ldots$ \\
\hline 5 & .6268 & Yes & Yes & Fuchsian \\
\hline
\end{tabular}

Theorem 5.12. If $G$ is a Kleinian group which contains a simple elliptic of order 4 or 5 , then

$$
\operatorname{Vol}\left(\mathbb{H}^{3} / G\right)>\operatorname{Vol}\left(\mathbb{H}^{3} / G_{(3,5,3)}=0.039 \ldots\right.
$$

Proof. First note that the unique arithmetic Kleinian group of minimal co-volume (the group $G_{(3,5,3)}[6]$ ) does not contain a simple elliptic element of order 4 or 5 . It is therefore clear from Table 2 that if $G$ is a Kleinian group containing an elliptic element $g$ of order 5 , then that group is either arithmetic or $\operatorname{ax}(g)$ has a precisely invariant collar of radius at least 0.6 . The result then follows from Lemma 5.11.

The case of a simple elliptic $g$ of order 4 is a little more complicated. We may assume that $G$ acts co-compactly and is not arithmetic. Next 
Table 1 implies that $G$ is either arithmetic or $\operatorname{ax}(g)$ has a precisely invariant collar of radius at least 0.534 except in the case that $G$ contains one of the groups $G_{4,10}$ or $G_{4,11}$ as a subgroup.

In the group $G_{4,10}$ the two closest elliptics of order 4 have parallel axes and therefore generate a Fuchsian group which is easily seen to be the $(4,4,5)$-triangle group. In the group $G_{4,11}$ the axes of the two closest elliptics of order 4 are perpendicular. But then a simple calculation shows the group generated by one of these elliptics and its conjugate by the other elliptic is the group $G_{4,10}$. Therefore we may suppose that $G$ contains the $(4,4,5)$-triangle group. Indeed since we may double the volume estimate unless there is a Klein 4-group on the boundary of this maximal collar, it is not difficult to see that we may assume the group $G$ contains the $(2,4,5)$-triangle group as a subgroup. In that case it is possible to establish a large volume bound along the lines of [26]. However we prefer to use the following more direct approach.

Now $\operatorname{ax}(g)$ has a precisely invariant collar of radius 0.5306 which contributes a volume of at least $0.0386 \ldots$. Next let $h$ denote an elliptic of order 5 in $G$. If $h$ is simple with respect to $G$, then we are done by virtue of what we first proved. Hence we may suppose otherwise and assume that $G$ contains an icosahedral subgroup $F$. Among the conjugacy classes of $F$ choose one whose finite fixed point, say $x_{0}$, is as close to $\operatorname{ax}(g)$ as possible. This is possible since $G$ acts co-compactly. Notice that $x_{0}$ cannot lie on $\operatorname{ax}(g)$. Now as $g$ has order 4 the hyperbolic line $\ell$ between $g^{2}\left(x_{0}\right)$ and $x_{0}$ passes through $\operatorname{ax}(g)$. Since $g$ is a simple elliptic, the line $\ell$ cannot be the axis of an elliptic of order 3 or 5 . Therefore according to Theorem 3 of $[12] \cosh \left(\rho\left(x_{0}, g^{2}\left(x_{0}\right)\right) \geq 2+\sqrt{5}\right.$. In particular this implies $\rho\left(x_{0}, \operatorname{ax}(g)\right) \geq \operatorname{arccosh}(2+\sqrt{5}) / 2 \approx 1.0613 \ldots$. Also by $[12$, Theorem 3$]$ the point $x_{0}$ is the center of a precisely invariant ball of radius $\operatorname{arccosh}((2+\sqrt{5}) / 2) / 2 \approx 0.6913 \ldots$. Thus the translates of the ball of radius $r_{0}=.53$ about $x_{0}$ do not meet the precisely invariant collar about $\operatorname{ax}(g)$. The stabilizer of this ball is the icosahedral group of order 60 . Therefore this precisely invariant ball contributes at least $\pi\left(\sinh \left(2 r_{0}\right)-2 r_{0}\right) / 60 \geq 0.01$ to the volume of the orbit space in addition to that given by the collar. Hence the orbit space has volume at least 0.48 which is greater than $0.03905 \ldots$ q.e.d.

Remark. Of course the methods above provide volume bounds when there is a simple elliptic axis of order 3 . However the bounds they do provide are not sufficient to deduce that the minimal co-volume Kleinian group does not contain a simple elliptic element of order 3 . 
Other techniques can be used in conjunction with these ideas to improve these bounds. We shall report on this elsewhere. We observe the following corollary which is a consequence of Theorem 5.9 and Theorem 5.12 .

Corollary 5.14. A Kleinian group of minimal co-volume does not contain a simple elliptic element of order greater than three.

\subsection{Symmetric hyperbolic 3 -manifolds}

As one further application of our methods we give volume bounds for the class of fairly symmetric 3 -manifolds. This term was coined by Kuiper [25] for those manifolds admitting a large cyclic symmetry with nonempty fixed point set. For the purposes of our discussion we define a symmetry of order $p$ of a hyperbolic 3 manifold $M$ to be an isometry of $M$ which is orientation preserving, has nonempty fixed point set and period $p$.

Theorem 5.15. Let $M^{3}$ be a closed hyperbolic 3-manifold admitting a symmetry of order $p \geq 4$. Then

$$
\operatorname{Vol}\left(M^{3}\right) \geq \nu(p)
$$

where

$$
\nu(p)= \begin{cases}0.81 & \text { if } p=4 \\ 1.08 & \text { if } p=5 \\ 1.40 & \text { if } p=6 \\ \left.2 p \mathcal{V}\left(r_{p}\right)\right) & \text { if } p \geq 7\end{cases}
$$

where $r_{p}$ is defined at (5.7).

Proof. Let $G$ be the fundamental group of $M^{3}$ acting as isometries on $\mathbb{H}^{3}$. Then $M^{3}=\mathbb{H}^{3} / G$. Let $f$ denote a lift of the given symmetry. Then $G_{1}=\langle G, f\rangle$ is a Kleinian group, $f$ is simple and the only primitive torsion in $G_{1}$ has order $p$. Let $r$ be the radius of a precisely invariant collar about $\operatorname{ax}(f)$. We have

$$
\operatorname{Vol}\left(\mathbb{H}^{3} / G_{1}\right) \geq 2 \mathcal{V}(r)
$$

by Theorem 4.9 and clearly

$$
\operatorname{Vol}\left(\mathbb{H}^{3} / G\right) \geq p \operatorname{Vol}\left(\mathbb{H}^{3} / G_{1}\right) .
$$

The result then follows in the case $p \geq 6$. For the case $p=4$, Table 1 implies that the group is arithmetic or contains an element of order 
5 unless $r>0.6130$. Now $\mathcal{V}(0.613)=0.104 \ldots$ and the smallest volume arithmetic manifold is known to be the Weeks manifold of volume $0.94 \ldots$ The result follows in this case.

For the case $p=5$, Table 2 implies the group is arithmetic unless $r>$ 0.626. Now $\mathcal{V}(0.626)=0.108 \ldots$, and in each case the arithmetic group in question contains the icosahedral group, and therefore the index of a torsion free subgroup is at least 60 . The result follows in this case too. q.e.d.

Remark. The Mennicke manifolds $M(p)$ are hyperbolic 3-manifolds whose fundamental group is isomorphic to the Fibonacci group $F(2,2 p)$. $M(p)$ is actually an $p$-fold covering of the sphere branched over the figure eight knot complement $\mathbb{S}^{3} \backslash \mathcal{K} . M(p)$ admits a cyclic isometry of order $2 p$ but it is not orientation preserving. There is a cyclic symmetry with fixed point of order $p$. Thus

$$
\operatorname{Vol}(M(p)) / p \rightarrow \operatorname{Vol}\left(\mathbb{S}^{3} \backslash \mathcal{K}\right)=2 T
$$

as $p \rightarrow \infty$. Here $T=1.015 \ldots$ is the volume of an ideal tetrahedron. Next we see from Lemma 3.25 that

$$
2 p \mathcal{V}\left(r_{p}\right) \approx p \sqrt{3} / 2
$$

as $p \rightarrow \infty$. Thus we find that Theorem 5.15 is off by a factor of $4 T / \sqrt{3}=$ $2.34 \ldots$ for large $p$.

\section{References}

[1] C. Adams, The noncompact hyperbolic 3-manifold of minimal volume Proc. Amer. Math. Soc. 100 (1987) 601-606.

[2] Limit volumes of hyperbolic three-orbifolds J. Differential Geom. 34 (1991) $115-141$.

[3] A. F. Beardon, The geometry of discrete groups, Springer, New York, 1983.

[4] A. Borel, Commensurability classes and volumes of hyperbolic 3-manifolds, Ann. Scuola Norm. Sup. Pisa Cl. Sci. 8 (1981) 1-33.

[5] C. Cao, F. W. Gehring \& G. J. Martin, Lattice constants and a lemma of Zagier, Contemporary Math. 211 (1997) 107-120.

[6] T. Chinburg \& E. Friedman, The smallest arithmetic hyperbolic three-orbifold, Invent. Math. 86 (1986) 507-527. 
[7] T. Chinburg, E. Friedman, K. N. Jones \& A. W. Reid, The arithmetic hyperbolic 3-manifold of smallest volume, in preparation.

[8] M. D. E. Conder \& G. J. Martin, Cusps, triangle groups and hyperbolic 3-folds, J. Austral. Math. Soc. Ser. A 55 (1993) 149-182.

[9] M. Culler \& P. Shalen, Paradoxical decompositions, 2-generator Kleinian groups, and volumes of hyperbolic 3-manifolds, J. Amer. Math. Soc. 5 (1992) 231-288.

[10] - The volume of a hyperbolic 3-manifold with Betti number 2, Preprint.

[11] - Volumes of hyperbolic Haken Manifolds I, Preprint.

[12] D. A. Derevnin \& A. D. Mednykh, Geometric properties of discrete groups acting with fixed points in Lobachevsky space, Soviet Math. Dokl. 37 (1988) 614-617.

[13] D. Gabai, On the geometric and topological rigidity of hyperbolic 3-manifolds, Ann. of Math., to appear.

[14] D. Gabai, R. Meyerhoff \& N. Thurston, Homotopy hyperbolic 3-manifolds are hyperbolic, to appear.

[15] F. W. Gehring \& G. J. Martin, Axial distances in discrete Möbius groups, Proc. Nat. Acad. Sci. U.S.A. 89 (1992) 1999-2000.

[16] , 6-torsion and hyperbolic volume, Proc. Amer. Math. Soc. 117 (1993) $727-735$.

[17] - Commutators, collars and the geometry of Möbius groups, J. Analyse Math. 63 (1994) 175-219.

[18] On the minimal volume hyperbolic 3-orbifold, Math. Res. Letters 1 (1994) $107-114$.

[19] _ Inequalities for Möbius transformations and discrete groups, J. Reine Angew. Math., 418 (1991) 31-76.

[20] - Commutator spectra for discrete groups with an elliptic generator, in preparation.

[21] - The volume of hyperbolic 3-folds with p-torsion, $p \geq 6$, Quart. J. Math., to appear.

[22] - Tetrahedral, Octahedral and Icosahedral points in a discrete group, to appear.

[23] F. W. Gehring, C. Maclachlan, G. J. Martin \& A.W. Reid, Arithmeticity, discreteness and volume, Trans. Amer. Math. Soc. 349 (1997) 3611-3643.

[24] T. Jørgensen, On discrete groups of Möbius transformations, Amer. J. Math. 98 (1976) $739-749$.

[25] N. Kuiper, Fairly symmetric manifolds, Preprint, Inst. Hautes Études Sci. 1988/89. 
[26] G. J. Martin, Triangle subgroups of Kleinian groups, Comment Math. Helv. 71 (1996) 339-361.

[27] B. Maskit, Kleinian groups, Springer, Berlin, 1987.

[28] R. Meyerhoff, The cusped hyperbolic 3-orbifold of minimum volume, Bull. Amer. Math. Soc. 13 (1985) 154-156.

[29] A lower bound for the volume of hyperbolic 3-manifolds, Canad. J. Math. 39 (1987) 1038-1056.

[30] J.W. Morgan \& H. Bass, The Smith Conjecture, Academic Press, New York, 1984.

[31] G. D. Mostow, Quasiconformal mappings in n-space and the rigidity of hyperbolic space forms, Inst. Hautes Études Sci. Publ. Math. 34 (1968) 53-104.

[32] W. P. Thurston, The geometry and topology of three-manifolds, Lecture Notes, Princeton University, 1976.

UNIVERSITY OF MICHIGAN

University of Auckland, New Zealand 